\title{
Progress in Molecular Dynamics Simulations of Gram- negative Bacterial Cell Envelopes
}

\author{
Alister Boags ${ }^{1 \ddagger}$, Pin-Chia Hsu ${ }^{1+}$, Firdaus Samsudin ${ }^{1 \ddagger}$, Peter J Bond ${ }^{2,3}$ and Syma Khalid ${ }^{1 *}$. \\ ${ }^{1}$ School of Chemistry, University of Southampton, Southampton, UK, SO17 1BJ. \\ ${ }^{2}$ Bioinformatics Institute (BII), Agency for Science, Technology and Research (A*STAR), Matrix 07-01, 30 Biopolis Street, \\ 138671 Singapore. \\ ${ }^{3}$ Department of Biological Sciences, National University of Singapore, 14 Science Drive 4, 117543 Singapore.
}

\section{Supporting Information Placeholder}

\begin{abstract}
Bacteria are protected by complex molecular architectures known as the cell envelope. The cell envelope is composed of regions with distinct chemical compositions and physical properties; namely membranes and a cell wall. To develop novel antibiotics to combat pathogenic bacteria, molecular level knowledge of the structure, dynamics and interplay between the chemical components of the cell envelope that surrounds bacterial cells is imperative. In addition, conserved molecular patterns associated with the bacterial envelope are recognized by receptors as part of the mammalian defensive response to infection, and an improved understanding of bacteria-host interactions would facilitate the search for novel immunotherapeutics. This Perspective introduces an emerging area of computational biology; multiscale molecular dynamics simulations of chemically complex models of bacterial lipids and membranes. We discuss progress to date, and identify areas for future development that will enable the study of aspects of the membrane components that are as yet unexplored by computational methods.
\end{abstract}

Bacteria are divided into two categories, Gramnegative and Gram positive, both of which include pathogens that are harmful to humans. Gram-negative bacteria have cell envelopes composed of two membranes, separated by a region known as the periplasm. The outer membrane (OM) is asymmetric in nature; the two leaflets differ in their compositions. The inner membrane contains a symmetric arrangement of phospholipids. In contrast, Gram-positive bacteria contain only one membrane, which is similar in composition to the inner membrane of Gram-negative bacteria. Both types of bacteria have a cell wall, which is composed of the biopolymer peptidoglycan. The combination of membrane plus cell wall gives rise to the characteristic semi-permeable properties of the cell envelope. To be effective, antibiotics must either cause bacterial cell death or inhibit cell growth. In both cases they must interact with the cell envelope, as they must either (i) disrupt the cell envelope, such that the cell contents leak out, or (ii) cross the cell envelope to gain access to the interior of the cell, where they may interfere with essential cellular process such as DNA replication and metabolism. The emergence of antimicrobial resistance is recognized as a major threat to human health. ${ }^{1}$ It is thus imperative to have a detailed knowledge of the structure-dynamics-function relationships of the cell wall and membranes, in order to develop new antibiotics under reduced pressure of resistance. Furthermore, molecules derived from the cell membrane and wall are utilized by the mammalian innate immune system to mount a defensive response. ${ }^{2}$ Over-amplification of such pathways can lead to sepsis, which remains the primary cause of death due to infection, highlighting the need for an improved understanding of the molecular mechanisms of immunostimulation. Due to the numerous molecular components involved, studying biological membranes in an in vivo condition remains an immensely challenging task. It is noteworthy that simulation of more complex biological membrane models is perhaps more prevalent for eukaryotic membranes, than bacterial ones, largely due to the simpler lipids in the former. In particular, both Marrink and Sansom and their research teams have independently reported eukaryotic membranes composed of multiple protein and lipid types. ${ }^{3,4}$ In recent years, computational modelling has provided useful insights into the behaviour of bacterial lipids and proteins in their native-like environment. Wonpil et al. recently reviewed current efforts in modelling and simulation of the bacterial OM, highlighting the dynamics of interactions between the components. ${ }^{5}$ Here we discuss recent progress in the molecular simulation, in both atomic-resolution and simplified 
coarse-grained (CG) representations, of the envelopes of Gram-negative and their component molecules.

\section{Gram-negative bacteria: the inner membrane}

The two leaflets of the inner membrane are primarily composed of a mixture of zwitterionic (phosphatidylethanolamine - PE) and anionic (cardiolipin - CL, phosphatidylglycerol - PG) phospholipids, for E. coli they are in the ratio, 90:5:5.

Simulation studies have traditionally employed one type of phospholipid to mimic the entire bilayer, with a greater emphasis placed on accurately modeling the membrane protein/peptide/drug molecules that may interact with the membrane. In the past few years, the importance of the heterogeneity of the phospholipid composition has become increasingly apparent, and now often a mixture of phospholipids is used in simulation studies $^{7,8}$. For example, Sansom \& co-workers simulated the UraA $\mathrm{H}^{+}$-uracil symporter from $E$. coli in a model of the inner membrane composed of a mixture of 1palmitoyl 2-oleoyl-phosphatidylethanolamine (POPE), 1-palmitoyl 2-oleoyl-phosphatidylglycerol (POPG); and CL (Figure 1). Despite the model membrane containing only $5 \% \mathrm{CL}$, preferential interaction of the protein with this lipid was observed. Simulations identified three specific cardiolipin binding sites on UraA, which led the authors to hypothesize that cardiolipin may form a 'proton-trap' that channels protons to and from the protein.

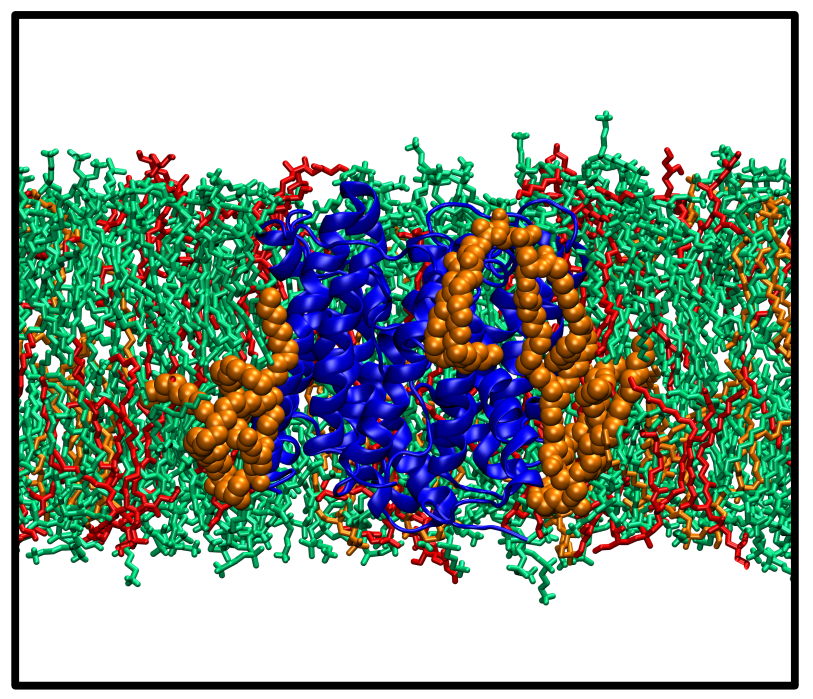

Figure 1. UraA symporter inserted in a POPE/POPG/CL bilayer ${ }^{3}$. UraA is shown in blue. The POPE, POPG and CL lipids are shown in green, red and orange, respectively. The three CL molecules that occupy the predicted CL binding sites are shown in orange $\mathrm{VdW}$. The water and ions are omitted for clarity.

The action of antimicrobial peptides at the inner membrane has been studied using atomistic molecular dynamics simulations. For example Amos et al (REF: Sci Rep. 2016 Nov 22;6:37639. doi: 10.1038/srep37639) compared two helical antimicrobial peptides; pleuro- cidin and magainin 2 in inner membrane mimetic bilayers composed of a mixture of zwitterionic and ionic lipids. They showed that pleurocidin has greater conformational flexibility and predicted that this was the basis of its greater potency against Gram-negative bacteria compared to magainin 2 , which has less conformational lability.

It is important to note here that simulation studies of complex symmetrical membranes also focus on the membranes of Gram-positive bacteria, mitochondria, plants and eukaryotes. ${ }^{3,4,-11}$ These studies are important in the progress in simulations of biological membranes, but are outside the scope of the current review, in which our focus is on Gram-negative bacteria.

\section{Gram-negative bacteria: the outer membrane}

The two leaflets of the outer membrane (OM) are rather different; the outer leaflet is composed of lipopolysaccharide (LPS) molecules, whereas the inner leaflet contains a mixture of zwitterionic and anionic phospholipids. In E. coli the inner leaflet of the outer membrane is composed of PE (90\%), PG (5\%) and CL (5\%). ${ }^{6}$ Thus, simplification of membrane models to one type of phospholipid is even more far removed from reality for the outer membrane than it is for the inner membrane. LPS molecules are large and heterogeneous, composed of the multiply-acylated lipid A "membrane anchor", which contains a phosphorylated $\beta(1 \rightarrow 6)$ diglucosamine unit, attached to an extended polysaccharide that includes core regions and $\mathrm{O}$-antigen. They also display two distinct phenotypes, i.e. smooth and rough; the former is characterized by the presence of a full-length $\mathrm{O}$-antigen, while the latter represents a truncated or the complete absence of the O-antigen. LPS, also known as endotoxin, provides structural integrity and chemical protection to the bacterial cell, and elicits strong immunological responses when invading mammalian hosts. In recent times, atomistic models of LPS of varying complexity have been reported in the literature, ${ }^{12,13}$ with new $C G$ models also emerging within the past few years. ${ }^{14-16}$ These models have increased the scope of simulation studies, such that bacterial membrane models now incorporate important chemical details representing the in vivo membrane environment. The first simulation of a membrane containing LPS in the literature was that of rough PA01 LPS chemotype from Pseudomonas aeruginosa reported by Lins et al. ${ }^{12}$ This was followed up by a study of the protein OprF embedded within an asymmetric outer membrane model containing rough LPS in the outer leaflet and PE in the inner leaflet. ${ }^{17}$ Recently, more detailed models of the E. coli outer membrane with additional anionic phospholipids like PG and CL in the inner leaflet have been reported. ${ }^{18-21}$ Simulations of outer membrane proteins (OMPs) in these detailed membranes have revealed protein-lipid interactions that are predicted to be key for the correct functioning of the protein. For example, the large extracellular loops of the 
TonB dependent transporter FecA were shown to specifically interact with LPS (Figure 2). ${ }^{13}$ These interactions altered both the short-term fluctuations in the position of the loops, and also the longer-term loop conformations. Two different mechanisms were reported for these effects; short-lived but frequently formed non-specific hydrogen-bonding interactions that alter the local fluctuations in loop movement, and secondly the bulky LPS molecules provide a steric resistance to larger conformational motions of the loops. Previous simulation studies of TonB-dependent transporters were all performed with simple phospholipid membranes and thus unable to predict these functionally important membrane-protein interactions. In addition to native OMPs, insights into the interaction of antimicrobial peptides (AMPs) and a range of small chemicals with the outer membrane have also been studied at the atomistic level. ${ }^{22-25}$ For example, extensive simulations were used to study the possible antimicrobial mechanisms of polymyxin B1 in both the IM and OM of E. coli, highlighting that AMPs are likely to interact with the two membranes in distinct ways. $^{24}$

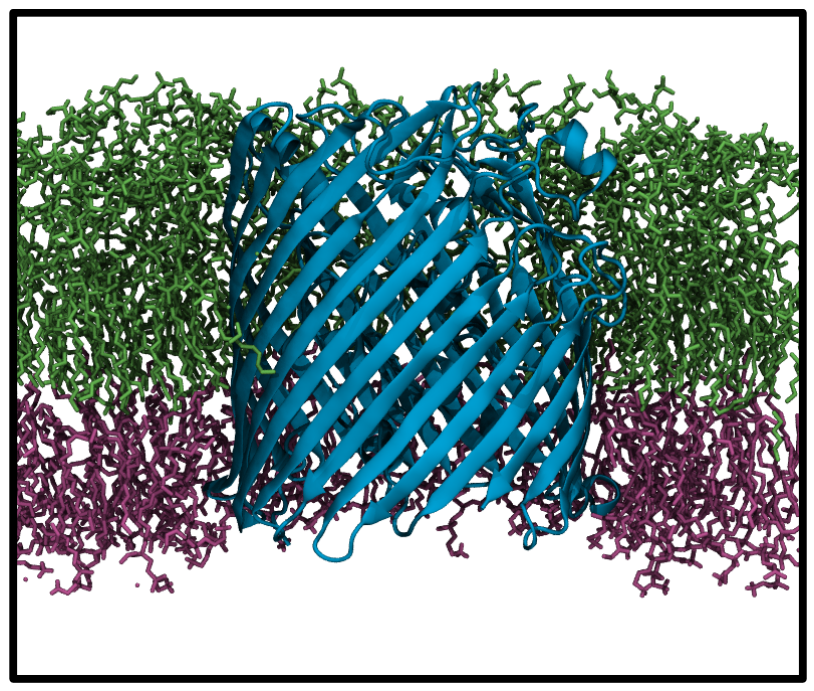

Figure 2. A snapshot of the FecA protein embedded in a model outer membrane, from Piggot et al. ${ }^{13} \mathrm{FecA}$ is shown in cyan, phospholipids are mauve and lipopolysaccharide molecules are lime green. Water and ions are omitted for clarity.

The structure of lipid A molecules varies from bacterial species to species, both in terms of the number and length of acyl chains, phosphorylation and presence of other functional modifications on the sugar units. Chemical modification can occur under certain environmental conditions, and many of these play a role in bacterial pathogenesis and immunoevasion. To date, atomicresolution simulations of lipid A from 12 different species of Gram-negative bacteria have been reported, as well as synthetic lipid A mimics developed for their potential therapeutic properties. ${ }^{26,27} \mathrm{Im}$ and co-workers provided a comparison of the properties of bilayers composed of several different lipid A types. ${ }^{28}$

At the CG level, we are only aware of models of LPS molecules developed within the framework of the popular MARTINI force-field. ${ }^{14}$ In one recent study, the ability of pristine fullerenes to penetrate the outer membrane was found to be dependent upon the presence of phospholipids in the outer leaflet as well as the inner leaflet. This raises an important question; how much phospholipid is really present in the outer leaflet of Gramnegative bacteria? At the present time, there is no definitive answer to this question, yet the answer will be crucial for a full understanding of the permeability of these membranes and thus we are reliant upon experimental colleagues for data to enable us to construct more accurate models. A related and equally important question is: how crowded is the outer membrane? This leads onto uncertainties regarding how much 'free' lipid there is in the outer membrane - in other words, how much lipid is dissociated from protein at any given time? From this we may also ask, how fluid really is the outer membrane? Addressing these questions requires complex simulations, to probe patterns of diffusion of proteins and lipids for which simplified, CG methods are ideal. Goose et al reported one of the first large-scale simulations of membranes that simultaneously incorporated many OMPs. ${ }^{29}$ Multiple copy numbers of the proteins OmpA, OmF, FhuA, LamB and NanC were simulated in mixtures of POPG and POPE phospholipids to mimic the outer membrane (CG models of LPS were not available at this time). The phospholipid diffusion around each protein was found to vary based on distance from protein, i.e. slower nearer to the protein. Furthermore as the membrane models incorporated increasing numbers of proteins, thereby making them more crowded, lateral diffusion of both proteins and lipids slowed down. Vattulainen and co-workers had previously reported similar trends for eukaryotic proteins, ${ }^{30}$ whilst Holdbrook et al extended the observations to a diverse range of vesicles with varying composition to show that lipid dynamics can be coupled to proteins as far as $6 \mathrm{~nm}$ apart, providing evidence for long-range membrane communication. ${ }^{31}$ Similarly, Rassam et al. employed large-scale coarse-grained simulations of BtuB and OmpF, along with fluorescence measurements, to assess protein-protein interactions in a crowded environment and their role in OM protein turnover in E. coli. ${ }^{32}$

\section{The bacterial cell wall.}

The cell wall of Gram-negative and Gram-positive bacteria is made up of a mesh of peptidoglycan, which is a sugar-peptide polymer. The glycan strand is conserved across all bacteria. In E. coli the sequence of the peptide stem of peptidoglycan is L-Ala (1) D-isoGlu (2) mA2pm (3) D- Ala (4) D-Ala (5), where m-A2pm is meso-diaminopimelic acid, a derivative of lysine. ${ }^{33}$ The width of this peptidoglycan layer in Gram-positive bac- 
teria is generally much thicker $(20-35 \mathrm{~nm})$ than in Gramnegative bacteria $(2-7 \mathrm{~nm}))^{34,35}$ In the latter it is found in the periplasm, sandwiched between the two membranes, whereas in the former it is found outside the cell membrane. The precise three-dimensional structure of peptidoglycan varies between species. There have been far fewer simulation studies of peptidoglycan compared to bacterial membranes owing to the lack of structural data for these molecules and their higher-order architecture, as well as difficulties inherent to modelling extended, branched carbohydrates. However, significant recent progress has been made in this area. Gumbart et al reported atomistic simulations of peptidoglycan from $E$. coli. $^{36}$ A single layer of peptidoglycan was constructed such that cross-linking between the peptidoglycan strands did not result in a highly ordered system, unlike those observed in previous models. ${ }^{33}$ The peptidoglycan layer was in good agreement with experimentally measured physical properties such as thickness, elasticity and pore dimensions, and thus provided confidence in the use of atomistic simulations for modeling the periplasm. We have recently simulated an integrative model of the full-length E.coli OMP OmpA in its dimeric form ${ }^{18,37}$ in the presence of a patch of OM and cell wall. ${ }^{19}$ A peptidoglycan fragment was docked into the conserved binding site of the globular, OmpA-like C-terminal domain, using as a template the homologous protein domain from A. baumanii, for which an X-ray structure cocrystallized with a peptidoglycan diaminopimelate has been solved. ${ }^{38}$

Simulations revealed the interaction between peptidoglycan and the protein to be rather labile, but persistent, and indicated how the domains of OmpA may serve as flexible anchors to provide adaptive mechanical support to the bacterial cell (Figure 3). These insights may offer alternative routes to the design of novel antibiotics.

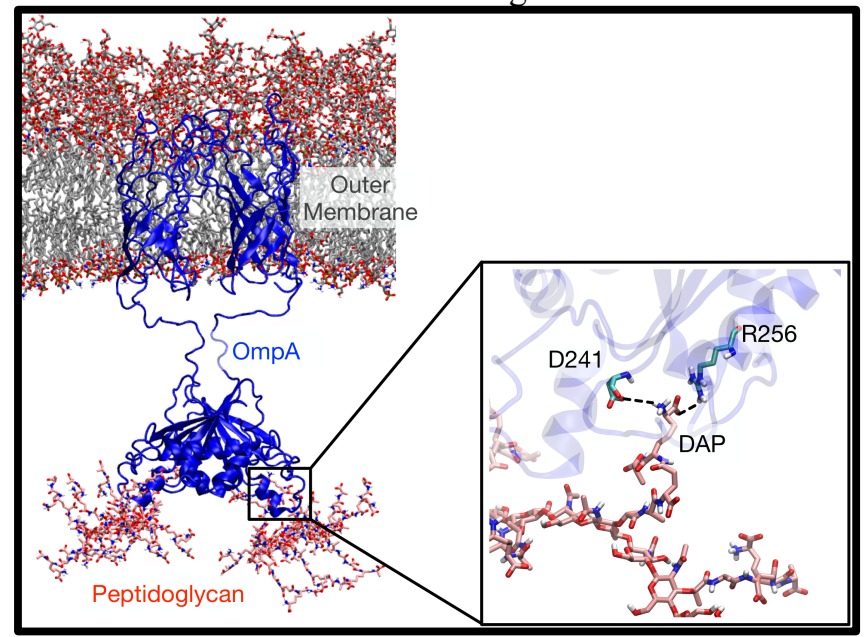

Figure 3. A model of peptidoglycan strands bound to a full-length OmpA homodimer embedded within a realistic outer membrane model containing LPS, as simulated by Samsudin et al. ${ }^{19}$ Enlarged image shows the salt bridge interactions between diaminopimelate (DAP) on the peptidoglycan with D241 and R256 residues on the OmpA Cterminal domain.

\section{Immunostimulation by components of the bacterial cell envelope.}

The evolutionarily conserved nature of the molecules associated with the bacterial cell wall and membrane and their absence in mammalian cells - mark them out as primary indicators of a new infection. The ten human members of the Toll-like receptor (TLR) family within the innate immune system are specialized for recognition of diverse pathogen-associated molecular patterns (PAMPs); bacterial PAMPs include e.g. cell wall peptidoglycan, and the flagellin protein of the bacterial flagellum. ${ }^{39}$ Another major PAMP is LPS, and its bioactive component lipid A, recognized by TLR4. This receptor has received particular interest over the years, not least because of its central role in propagation of endotoxic shock and sepsis during overwhelming Gram-negative bacterial infections. ${ }^{2}$ A specialized relay of proteins exist to extract and transfer LPS molecules from bacterial membranes (or aggregates thereof) to the terminal cell surface receptor, TLR4, in complex with its lipidbinding co-receptor, MD-2. The great stability of the bacterial $\mathrm{OM}$ represents an interesting challenge for successful host recognition. Recently, atomic-resolution simulations were used to rigorously calculate the affinity of individual lipid A molecules for the lipid bilayer environment; a large barrier to lipid A extraction exists as a consequence of its extreme hydrophobicity combined with the divalent cation cross-links between phosphorylated headgroups. ${ }^{27}$ Nevertheless, the MD-2 coreceptor was shown to have a comparable affinity for lipid A, revealing that the TLR4 system has evolved to create a membrane-like environment for endotoxin recognition. Furthermore, simulations revealed that the binding cavity of MD-2 exhibits clamshell-like dynamics, ${ }^{26}$ which allow it to locally adapt to the size and shape of bound ligand. These dynamics, also observed in related proteins, ${ }^{40,41}$ are allosterically coupled to the higher-order assembly of the TLR4/MD-2 complex, ${ }^{26}$ thus ensuring that activation of TLR4 via dimerization occurs only in the presence of agonistic molecules such as hexaacylated lipid A, but not antagonists such as lipid IVa, its biosynthetic precursor, or synthetic antagonists like Eritoran. ${ }^{27}$ These simulations provide a platform for prediction of the activities of novel immunotherapeutic compounds, towards treatment of sepsis and other inflammatory conditions associated with bacterial infection.

\section{What next for simulations of the bacterial cell en- velope?}

The last few years have seen tremendous progress in the complexity, time-scales, length-scales and therefore biological relevance of classical molecular simulation studies of components of the bacterial cell envelope. At 
the atomistic level, all of the components can now be simulated individually and in complex assemblies (Figure 4).

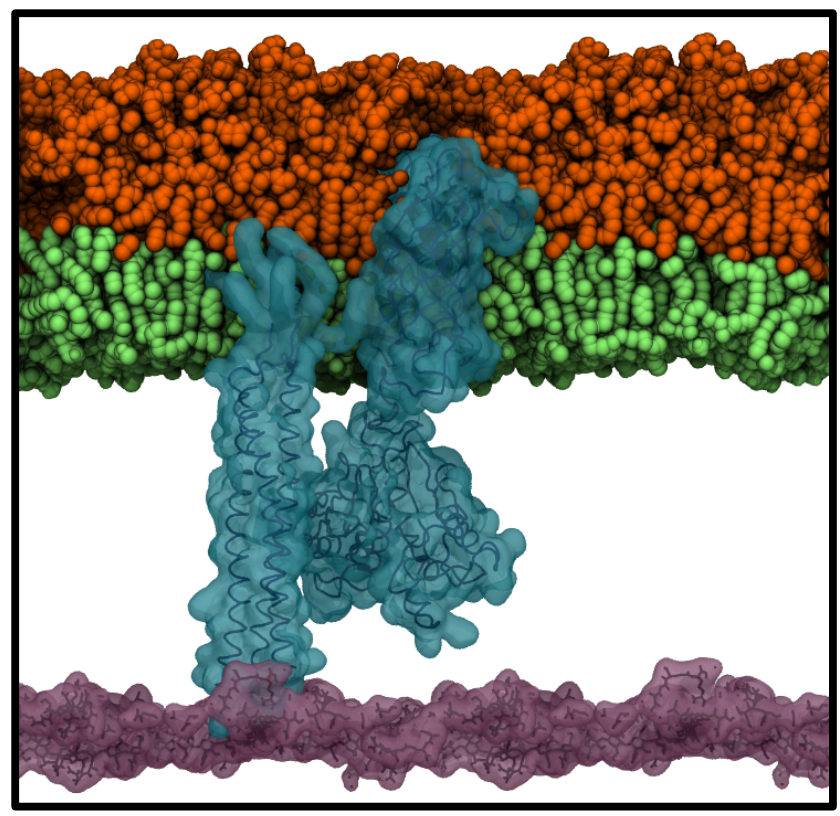

Figure 4. A Snapshot from an atomistic simulation of the OM (green and orange) containing the full length OmpA dimer (cyan, right). Braun's lipoprotein (cyan, left) is held in position via a lipid anchor in the OM and covalent attachment to a sheet of peptodoglycan (pink). This is an extension of the peptidoglycan model reported in Samsudin et $\mathrm{al}^{10}$.

However the biology of these systems is inherently multi-scale; there are time- and length-scales that are still beyond currently available models, if not methods. For example, it has long been speculated that some proteins and lipids are preferentially located at the poles of rod-shaped bacteria. To understand how and why, requires simulation of larger biological membrane systems (the dimension of $E$. coli is approximately $0.5 \times 2.0 \mu \mathrm{m}$ ) on relevant timescales, necessitating even coarsergrained models than those based on the $\sim 4: 1$ mapping that have recently become so popular. In addition, genome scale models of bacteria are also emerging which further expand the system sizes that are addressable. ${ }^{42,43}$ At the other end of the scale, to truly understand the chemical processes that can lead to the development of bacterial antibiotics resistance, such as the covalent modification of lipids by membrane enzymes, requires calculations at the quantum-level. For example it is known that the outer membrane enzymes PagP and PagL modify the number of lipid tails of the lipid A portion of LPS. ${ }^{44}$ Quantum mechanical (QM) calculations of membrane/protein systems are exceedingly rare, and have so far required a mixed $\mathrm{QM} /$ molecular mechanics (QM/MM) treatment. ${ }^{45}$ Traditionally this has in the most part been due to the larger system sizes and compositional complexity of membrane protein systems compared to their soluble protein counterparts. However, recent progress in $\mathrm{QM}$ codes, such as the development of linear scaling density functional codes that can handle biological molecules provide tremendous promise. ${ }^{46}$ Attention to the development and validation of appropriate protocols to add quantum-level detail to the current arsenal of classical simulation tools will undoubtedly herald a step-change in terms of computational studies of bacterial membranes, and concurrently, rational design of antibiotics and immunotherapeutics.

\section{AUTHOR INFORMATION}

\section{Corresponding Author}

S.Khalid@soton.ac.uk to whom correspondence should be addressed.

\section{Author Contributions}

$\ddagger$ These authors contributed equally.

\section{Funding Sources}

FS is funded by BBRSC grant number: BB/M029573

\section{ACKNOWLEDGMENT}

We thank Antreas Kalli for providing Figure 1.

\section{REFERENCES}

WHO. Antimicrobial Resistance: Global Report on Surveillance. Bull. World Health Organ. 2014, 61 (3), 383-394.

O'Neill, L. A. J.; Bryant, C. E.; Doyle, S. L.; Neill, L. A. J. O. Therapeutic Targeting of Toll-Like Receptors for Infectious and Inflammatory Diseases and Cancer. Pharmacol. Rev. 2009, 61 (2), 177-197.

Koldsø, H.; Sansom, M. S. P. Organization and Dynamics of Receptor Proteins in a Plasma Membrane. J. Am. Chem. Soc. 2015, 137 (46), 14694-14704.

van Eerden, F. J.; van den Berg, T.; Frederix, P. W. J. M.; de Jong, D. H.; Periole, X.; Marrink, S. J. Molecular Dynamics of Photosystem II Embedded in the Thylakoid Membrane. J. Phys. Chem. B 2016, acs.jpcb.6b06865.

Patel, D. S.; Qi, Y.; Im, W. Modeling and Simulation of Bacterial Outer Membranes and Interactions with Membrane Proteins. Curr. Opin. Struct. Biol. 2017, 43 (Figure 1), 1-17.

Lugtenberg, E. J. .; Peters, R. Distribution of Lipids in Cytoplasmic and Outer Membranes of Escherichia Coli K12. Biochim. Biophys. Acta 1976, 441 (195 6), 38-47. 
Kalli, A. C.; Sansom, M. S. P.; Reithmeier, R. A. F. Molecular Dynamics Simulations of the Bacterial UraA H+-Uracil Symporter in Lipid Bilayers Reveal a Closed State and a Selective Interaction with Cardiolipin. PLoS Comput. Biol. 2015, 11 (3), 127.

(8) Alcock, F.; Stansfeld, P. J.; Basit, H.; Habersetzer, J.; Matthew, A. B. Assembling the Tat Protein Translocase. Elife 2016, 5:e20718, 1-28.

Piggot, T. J.; Holdbrook, D. A.; Khalid, S. Electroporation of the E. Coli and S. Aureus Membranes: Molecular Dynamics Simulations of Complex Bacterial Membranes-Suppinfo. J. Phys. Chem. 2011.

(10) Rui, H.; Lee, K. Il; Pastor, R. W.; Im, W. Molecular Dynamics Studies of Ion Permeation in VDAC. Biophys. J.2011, 100(3), 602-610.

(11) Hedger, G.; Rouse, S. L.; Doma??ski, J.; Chavent, M.; Kolds??, H.; Sansom, M. S. P. Lipid-Loving ANTs: Molecular Simulations of Cardiolipin Interactions and the Organization of the Adenine Nucleotide Translocase in Model Mitochondrial Membranes. Biochemistry 2016, 55 (45), 62386249.

(12) Kirschner, K. N.; Lins, R. D.; Maass, A.; Soares, T. A. A Glycam-Based Force Field for Simulations of Lipopolysaccharide Membranes: Parametrization and Validation. J. Chem. Theory Comput. 2012, 8 (11), 4719-4731.

(13) Piggot, T. J.; Holdbrook, D. A.; Khalid, S. Conformational Dynamics and Membrane Interactions of the E. Coli Outer Membrane Protein FecA: A Molecular Dynamics Simulation Study. Biochim. Biophys. Acta. 2013, 1828 (2), 284-293.

(14) Hsu, P.-C.; Jefferies, D.; Khalid, S. Molecular Dynamics Simulations Predict the Pathways via Which Pristine Fullerenes Penetrate Bacterial Membranes. J. Phys. Chem. B 2016, 120 (43), 11170-11179.

(15) $M a, H . ;$ Irudayanathan, F. J.; Jiang, W.; Nangia, S. Simulating Gram-Negative Bacterial Outer Membrane: A Coarse Grain Model. J. Phys. Chem. B2015, No. 119, 14668-14682.

(16) Jefferies, D.; Hsu, P. C.; Khalid, S. Through the Lipopolysaccharide Glass: A Potent Antimicrobial Peptide Induces Phase Changes in Membranes. Biochemistry 2017, acs.biochem.6b01063.

(17) Straatsma, T. P.; Soares, T. A. Characterization of the Outer Membrane Protein OprF of
Pseudomonas Aeruginosa in $a$ Lipopolysaccharide Membrane by Computer Simulation. Proteins 2009, 74(2), 475-488.

(18) Ortiz-Suarez, M. L.; Samsudin, F.; Piggot, T. J.; Bond, P. J.; Khalid, S. Full-Length OmpA: Structure, Function, and Membrane Interactions Predicted by Molecular Dynamics Simulations. Biophys. J. 2016, $111(8), 1692-1702$.

(19) Samsudin, F.; Ortiz-Suarez, M. L.; Piggot, T. J.; Bond, P. J.; Khalid, S. OmpA: A Flexible Clamp for Bacterial Cell Wall Attachment. Structure 2016, 24(12), 1-9.

(20) Wu, E. L.; Fleming, P. J.; Yeom, M. S.; Widmalm, G.; Klauda, J. B.; Fleming, K. G.; Im, W. E. Coli Outer Membrane and Interactions with OmpLA. Biophys. J. 2014, 106(11), 2493-2502.

(21) Patel, D. S.; Re, S.; Wu, E. L.; Qi, Y.; Klebba, P. E.; Widmalm, G.; Yeom, M. S.; Sugita, Y.; Im, W. Dynamics and Interactions of OmpF and LPS: Influence on Pore Accessibility and lon Permeability. Biophys. J. 2016, 110(4), 930-938.

(22) Pino-Angeles, A.; Leveritt, J. M.; Lazaridis, T. Pore Structure and Synergy in Antimicrobial Peptides of the Magainin Family. PLoS Comput. Biol. 2016, 12(1), 1-17.

(23) Bemporad, D.; Luttmann, C.; Essex, J. W. Computer Simulation of Small Molecule Permeation across a Lipid Bilayer: Dependence on Bilayer Properties and Solute Volume, Size, and Cross-Sectional Area. Biophys. J. 2004, 87(1), 113.

(24) Berglund, N. A.; Piggot, T. J.; Jefferies, D.; Sessions, R. B.; Bond, P. J.; Khalid, S. Interaction of the Antimicrobial Peptide Polymyxin B1 with Both Membranes of E. Coli: A Molecular Dynamics Study. PLoS Comput. Biol. 2015, 11 (4), e1004180.

(25) Carpenter, T. S.; Parkin, J.; Khalid, S. The Free Energy of Small Solute Permeation through the Escherichia Coli Outer Membrane Has a Distinctly Asymmetric Profile. J. Phys. Chem. Lett. 2016, 3446-3451.

(26) Paramo, T.; Piggot, T. J.; Bryant, C. E.; Bond, P. J. The Structural Basis for Endotoxin-Induced Allosteric Regulation of the Toll-like Receptor 4 (tIr4) Innate Immune Receptor. J. Biol. Chem. 2013, 288(51), 36215-36225.

(27) Paramo, T.; Tomasio, S. M.; Irvine, K. L.; Bryant, C. E.; Bond, P. J. Energetics of Endotoxin Recognition in the Toll-Like Receptor 4 Innate Immune Response. Sci. Rep. 2015, 5(April), 17997. 
(28) Kim, S.; Patel, D. S.; Park, S.; Slusky, J.; Klauda, J. B.; Widmalm, G.; Im, W. Bilayer Properties of Lipid A from Various Gram-Negative Bacteria. Biophys. J.2016, $111(8), 1750-1760$.

(29) Goose, J. E.; Sansom, M. S. P. Reduced Lateral Mobility of Lipids and Proteins in Crowded Membranes. PLoS Comput. Biol. 2013, 9(4).

(30) Niemelä, P. S.; Miettinen, M. S.; Monticelli, L.; Hammaren, H.; Bjelkmar, P.; Murtola, T.; Lindahl, E.; Vattulainen, I. Membrane Proteins Diffuse as Dynamic Complexes with Lipids. J. Am. Chem. Soc. 2010, 132(22), 7574-7575.

(31) Holdbrook, D. A.; Huber, R. G.; Piggot, T. J.; Bond, $P$. J.; Khalid, S. Dynamics of Crowded Vesicles: Local and Global Responses to Membrane Composition. PLoS One 2016, 11 (6), e0156963.

(32) Rassam, P.; Copeland, N. a.; Birkholz, O.; Tóth, C.; Chavent, M.; Duncan, A. L.; Cross, S. J.; Housden, N. G.; Kaminska, R.; Seger, U.; et al. Supramolecular Assemblies Underpin Turnover of Outer Membrane Proteins in Bacteria. Nature 2015, 523, 333-336.

(33) Vollmer, W.; Blanot, D.; De Pedro, M. A. Peptidoglycan Structure and Architecture. FEMS Microbiol. Rev. 2008, 32(2), 149-167.

(34) Vollmer, W.; Seligman, S. J. Architecture of Peptidoglycan: More Data and More Models. Trends in Microbiol. 2010, 18(2), 59-66.

(35) Beeby, M.; Gumbart, J. C.; Roux, B.; Jenssen, G. J. Architecture and Assembly of the Gram-Positive Cell Wall. Mol. Microbiol. 2013 , 88(4), 664-672.

(36) Gumbart, J. C.; Beeby, M.; Jensen, G. J.; Roux, B. Escherichia Coli Peptidoglycan Structure and Mechanics as Predicted by Atomic-Scale Simulations. PLoS Comput. Biol. 2014, 10(2).

Marcoux, J.; Politis, A.; Rinehart, D.; Marshall, D. P.; Wallace, M. I.; Tamm, L. K.; Robinson, C. V. Mass Spectrometry Defines the C-Terminal Dimerization Domain and Enables Modeling of the Structure of Full-Length OmpA. Structure 2014, $22(5), 781-790$.
Kumarasiri, M.; Hesek, D.; Lee, M.; Mobashery, S.; Song, J. H.; Kim, S. l.; et al. Mechanism of Anchoring of OmpA Protein to the Cell Wall Peptidoglycan of the Gram-Negative Bacterial Outer Membrane. FASEB J. 2012, 26(1), 219-228.

(39) Berglund, N. A.; Kargas, V.; Ortiz-Suarez, M. L.; Bond, P. J. The Role of Protein-Protein Interactions in Toll-like Receptor Function. Prog. Biophys. Mol. Biol. 2015, 119(1), 72-83.

(40) Paramo, T.; East, A.; Garzón, D.; Ulmschneider, M. B.; Bond, P. J. Efficient Characterization of Protein Cavities within Molecular Simulation Trajectories: Trj-Cavity. J. Chem. Theory Comput. 2014, 10(5), 2151-2164.

(41) Ortiz-Suarez, M. L.; Bond, P. J. The Structural Basis for Lipid and Endotoxin Binding in RP105-MD-1, and Consequences for Regulation of Host Lipopolysaccharide Sensitivity. Structure 2016, 24(1), 200-211.

(42) Liu, J. K.; O'Brien, E. J.; Lerman, J. a; Zengler, K.; Palsson, B. O.; Feist, A. M. Reconstruction and Modeling Protein Translocation and Compartmentalization in Escherichia Coli at the Genome-Scale. BMC Sys. Biol. 2014, 8, 110.

(43) Zhuang, K.; Vemuri, G. N.; Mahadevan, R. Economics of Membrane Occupancy and RespiroFermentation. Mol. Sys. Biol. 2011 , 7(500), 500.

(44) Trent, M. S. Biosynthesis, Transport, and Modification of Lipid A. Biochem Cell Biol. 2004, 82(1), 71-86.

(45) Lonsdale, R.; Rouse, S. L.; Sansom, M. S. P.; Mulholland, A. J. A Multiscale Approach to Modelling Drug Metabolism by Membrane-Bound Cytochrome P450 Enzymes. PLoS Comput. Biol. 2014, 10(7).

(46) Skylaris, C. K.; Haynes, P. D.; Mostofi, A. A.; Payne, M. C. Introducing ONETEP: Linear-Scaling Density Functional Simulations on Parallel Computers. J. Chem. Phys. 2005, 122(8). 
Insert Table of Contents artwork here

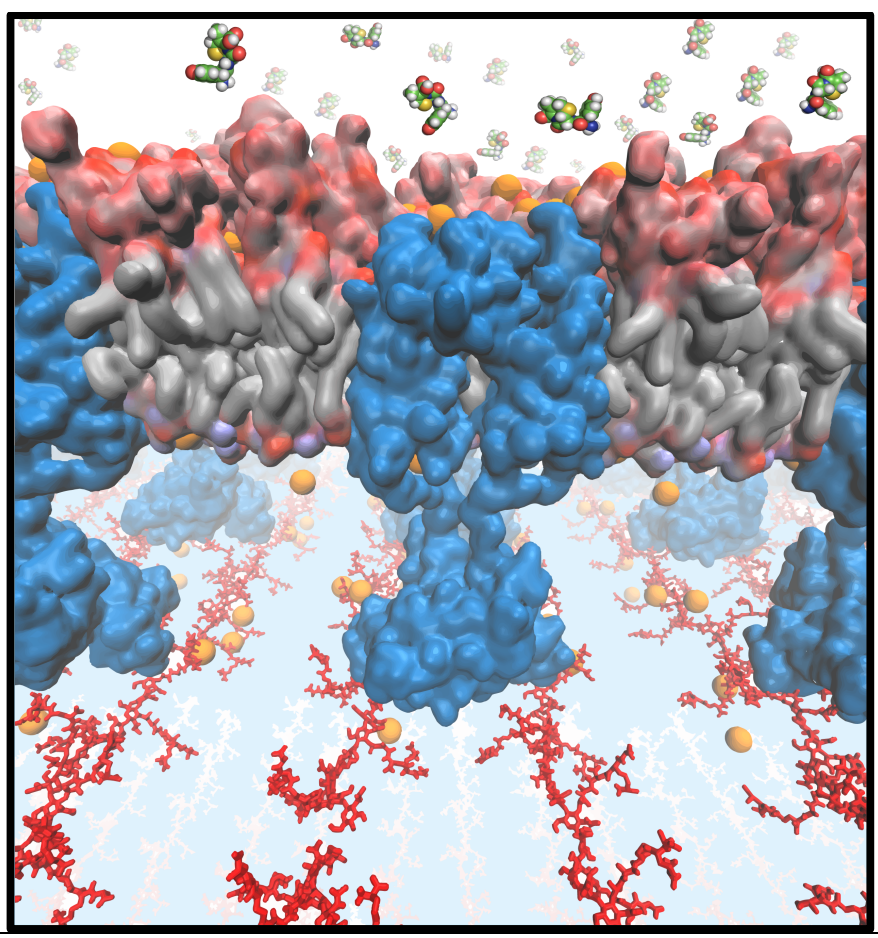

